\title{
Adaptive Neural Coding Dependent on the Time-Varying Statistics of the Somatic Input Current
}

\author{
Jonghan Shin \\ Christof Koch \\ Computation and Neural Systems Program, California Institute of Technology, Pasadena, \\ CA 91125, U.S.A.
}

\section{Rodney Douglas}

Institut für Neuroinformatik, UNI/ETH, Zürich, Switzerland

It is generally assumed that nerve cells optimize their performance to reflect the statistics of their input. Electronic circuit analogs of neurons require similar methods of self-optimization for stable and autonomous operation. We here describe and demonstrate a biologically plausible adaptive algorithm that enables a neuron to adapt the current threshold and the slope (or gain) of its current-frequency relationship to match the mean (or dc offset) and variance (or dynamic range or contrast) of the time-varying somatic input current. The adaptation algorithm estimates the somatic current signal from the spike train by way of the intracellular somatic calcium concentration, thereby continuously adjusting the neurons' firing dynamics. This principle is shown to work in an analog VLSI-designed silicon neuron.

\section{Introduction}

In the developing as well as in the mature animal, neuronal firing properties (or neural code) reflect the statistical properties of presynaptic neurons (Calvin, 1978; van Steveninck, Bialek, Potters, \& Calson, 1994; Smirnakis, Berry, Warland, Bialek, \& Meister, 1997). For instance, it has been argued that the most efficient representation of the input should use each firing rate with equal probability (Laughlin, 1981) or that the entropy of the firing rate should be maximized subject to some constraint, such as average firing rate (Baddeley et al., 1997). Spiking neurons might exploit a coding that is similar to that used in the class of one-bit analog-digital converters known as oversampled Delta-Sigma modulators (Wong \& Gray, 1990; Shin, Lee, \& Park, 1993). And these representations need to be invariant to environmental changes such as temperature, cell growth, channel turnover, and so on that affect neuronal performance. This raises the question of how a neuron maintains homeostasis in the face of a changing environment or a chang- 
ing input. An adaptive mechanism that continuously seeks some optimum within an allowed class of possibilities would give a superior performance compared to a neuron with a fixed input-output relationship (Widrow \& Stearns, 1985).

How can this goal be accomplished at the single-cell level? Many possibilities come to mind. Experimental evidence from neocortical cells implicates a change in the synaptic input that down- or upregulates their postsynaptic effect (Carandini \& Ferster, 1997; Turrigiano, Leslie, Desai, Rutherford, \& Nelson, 1998); this effect may be mediated by metabotropic receptors (Mclean \& Palmer, 1996). However, other biophysical or biochemical mechanisms are likely to be involved as well. Given the crucial role of free intracellular calcium in controlling activation of potassium-dependent conductances as well as a host of enzymes, $\mathrm{Ca}^{2+}$-binding proteins, and calciumsensitive genes, it is bound to be involved in maintaining homeostasis. Abbott and his colleagues (Abbott \& LeMasson, 1993; LeMasson, Marder, \& Abbott, 1993) were the first to propose a quantitative link between the mean intracellular, somatic calcium concentration-serving as an indicator for mean firing activity level-and the density of a calcium and a calcium-activated potassium conductance to achieve a criterion mean firing rate for the cell.

Electronic counterparts of biological neurons-so-called silicon neurons designed using integrated circuit technology (Mahowald \& Douglas, 1991) have to deal with a related problem: a very large number of associated parameters that need to be set properly in order for the cell to function properly (rate constants, peak conductances, and so on). And the performance of such neurons needs to be stable in the face of fluctuations of bias voltages, operating temperature, and transistor mismatch. Finally, the sensitivity of these neurons should also reflect changing input statistics. If we are ever going to operate large networks of VLSI neurons, we need to incorporate adaptation into the basic operation of each neuron (Douglas, Koch, Mahowald, \& Martin, 1999).

We here focus on the question of how the firing properties of a spiking neuron depend on the magnitude range change of the time-varying somatic current signals delivered by synaptic input or intracellular electrode to the soma. We do so on the basis of adaptive coding theory (Jayant \& Noll, 1984). The input current causes action potentials to be triggered. Signal estimation theory (Rieke, Warland, van Steveninck, \& Bialek, 1996; Gabbiani \& Koch, 1998) provides us with an estimation filter to infer the continuous input current from these discrete events. The filter provides an optimal estimate of the input in a least-square sense.

We argue that the intracellular free calcium concentration $\left[\mathrm{Ca}^{2+}\right]_{i}$ at the cell body represents such an estimation filter. Each action potential leads to an influx of calcium ions via high-threshold, voltage-dependent calcium channels. A variety of processes such as pumping, diffusion, and buffering cause $\left[\mathrm{Ca}^{2+}\right]_{i}$ to decay in time (Koch, 1998). We use $\left[\mathrm{Ca}^{2+}\right]_{i}$ to estimate the average input current and its standard deviation. These estimates control the amplitude of two conductances that affect the cell's discharge curve, 
enabling the range of the input signal to be optimally matched to the inputoutput function of the cell. Note that we are not arguing that the function of the neuron is to reconstruct its input but that an estimate of the cell's input can be used to adapt the neuron to the time-varying statistics of the somatic input current. This principle is implemented and shown to work in a real-time silicon pyramidal neuron (Mahowald \& Douglas, 1991).

\section{Methods}

We used our previously characterized silicon neurons (Mahowald \& Douglas, 1991; Douglas \& Mahowald, 1995, 1998) in this study. These artificial neurons emulate the electrophysiology of the somata of regular spiking neocortical pyramidal cells. The version used in this study comprised a single somatic compartment and a simple passive dendritic load. The somatic compartment includes five voltage-dependent currents as well as a leak current: the sodium spike current; the delayed rectifier potassium current; a transient, inactivating potassium current (A current); a calcium-dependent potassium current; a high-threshold calcium current; and the leakage current.

These currents can be approximated by a Hodgkin-Huxley-like formalism (Hodgkin \& Huxley, 1952),

$$
I(t)=\bar{g} \cdot m(t, V)^{i} \cdot h(t, V)^{j} \cdot(V-E),
$$

where $\bar{g}$ is the maximum conductance; $m$, the activation variable taken to the $i$ th power; $h$, the inactivation variable taken to the $j$ th power; $V$, the membrane potential; and $E$, the reversal potential of the current. The dynamics of each activation and inactivation particle is governed by the usual first-order differential equation (see the appendix).

The circuits of the silicon neuron approximate the effect of these equations, using transconductance amplifiers to emulate the voltage-dependent conductances, while follower integrators provide the necessary dynamics. The details of the analog VLSI circuit implementation of the HodgkinHuxley-like formalism are described elsewhere (Douglas \& Mahowald, 1995, 1998).

The silicon neuron contains circuitry for simulating intracellular calcium concentrations and the calcium-dependent potassium current (or afterhyperpolarization, AHP, current). The calcium concentration circuit emulates the intracellular, free calcium concentration with the aid of a capacitance in parallel with a resistance whose behaviors can be approximated using a first-order differential equation that lumps buffering, pumping, and diffusion of $\mathrm{Ca}^{2+}$ into a single decay term (Bower \& Beeman, 1995),

$$
\tau_{C a} \frac{d\left[C a^{2+}\right]_{i}}{d t}=-\left[C a^{2+}\right]_{i}+\kappa I_{C a}+C A R E F,
$$

where $\tau_{C a}$ is the time constant of calcium decay, $I_{C a}$ is the action-potential- 

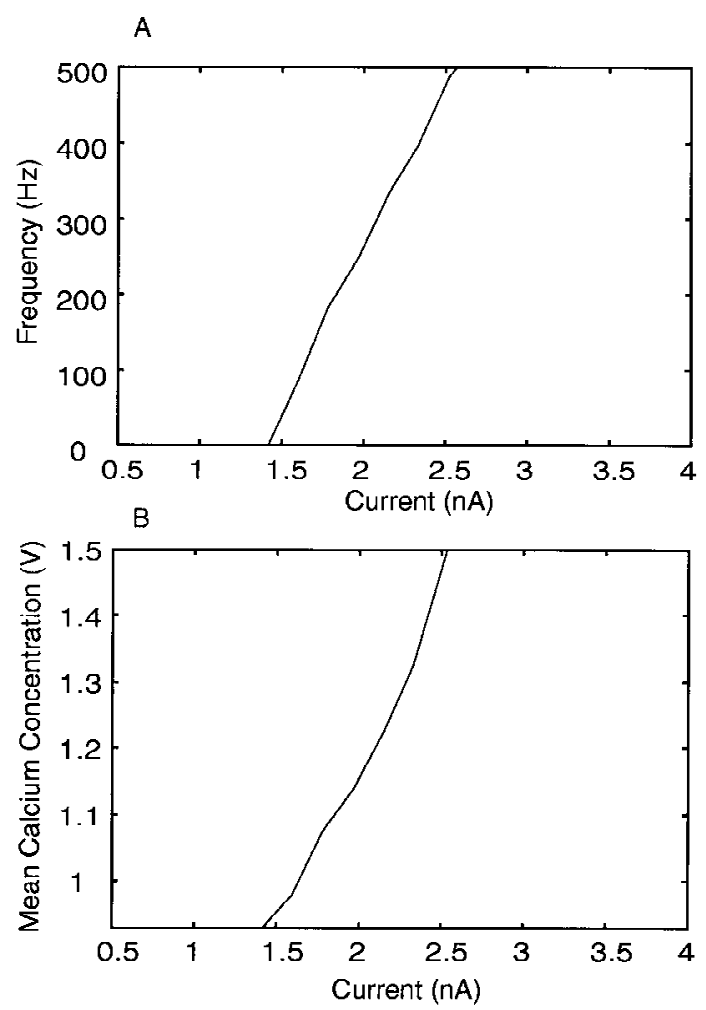

Figure 1: (A) The steady-state f-I curve and (B) mean intracellular calcium concentration versus input current curve of the silicon neuron for sustained current injections. The mean calcium concentration reflects the sustained somatic current level. Therefore, information about the mean somatic current signal can be estimated by the mean calcium concentration.

evoked $\mathrm{Ca}^{2+}$ current via a high-threshold, voltage-activated calcium conductance, $\kappa$ is a constant that converts the incoming calcium current into a concentration change, and CAREF is the resting calcium concentration. This element is followed by a calcium-dependent but voltage-independent activation variable $m$ determining the activation of the calcium-dependent potassium current.

Figures $1 \mathrm{~A}$ and $1 \mathrm{~B}$ plot the steady-state current-frequency relationship as well as mean intracellular calcium concentration-current relation of our silicon neuron in its standard settings. In this article, all figures were obtained with the same parameter settings of the chip, except the discharge curves II, III, II', and III' in Figure 7. 
The parameters that control the various currents are set as bias voltages on pads of the silicon chip. The voltages are provided by multiple digitalto-analog converters controlled by a digital computer. This machine also monitors the membrane potential $V$ and the low-pass filtered response of the spike-evoked $\mathrm{Ca}^{2+}$ signal at the soma and-on the basis of the adaptive neural coding procedure to be discussed below-sends back a bias voltage to adjust various circuit elements of the silicon neuron.

\section{Signal Reconstruction via Low-Pass Filtering}

Bayly (1968) theoretically showed (using a model equivalent to the integrateand-fire neuron model) that continuous signal reconstruction (or decoding) from spike trains can be accomplished by low-pass filtering. Shin, Lee, \& Park (1993) showed that better signal reconstruction (from a signal-to-noise ratio and entropy point of view) can be acquired by the same low-pass filtering (time constant: 5-20 msec) of spike trains from spiking neurons using the spectral noise shaping pulse coding principle. These methods can be implemented at either the single neuron level with the help of potassium currents (Shin, 1994) or at the network level via recurrent/feedback inhibition (Shin, 1996). It is expected that the nervous system takes advantage of this fact at its decoding sites, the dendritic membrane and intracellular calcium concentration. Indeed, the response of a synapse to an action potential usually shows the characteristics of a low-pass filter and is sometimes approximated by the response of an RC filter (Johnston \& Wu, 1995). Moreover, the low-pass filter characteristics of muscle in decoding efferent neural spikes is well known (Fatt \& Katz, 1951; Partridge, 1965).

Our adaptive neural coding procedure uses the low-pass filtered response of the spike-evoked $\mathrm{Ca}^{2+}$ signal to monitor the time-varying somatic current signal condition.

Figure 2 shows the dynamics of calcium buffering and extrusion following a single action potential, as emulated by the calcium concentration circuit of the silicon neuron. If the single action potential is described by a delta function, $\delta(t)$, then the impulse response of the calcium buffering and extrusion circuit is approximated (for $t>0$ ) as

$$
h(t)=\beta e^{-t / \tau_{C a}},
$$

where $\beta$ is the maximum magnitude of the observed impulse response (see Figure $2 \mathrm{~B}$ ) and $\tau_{\mathrm{Ca}}$ is the time constant of the calcium decay that can be electronically controlled. In other words, the calcium buffering and extrusion function implements a low-pass filter with a frequency-dependent gain of

$$
\text { Gain }=\frac{\beta \tau_{C a}}{\sqrt{\left(2 \pi f \tau_{C a}\right)^{2}+1}} .
$$

The $3 \mathrm{~dB}$ cutoff frequency, that is, the frequency for which the gain is reduced 

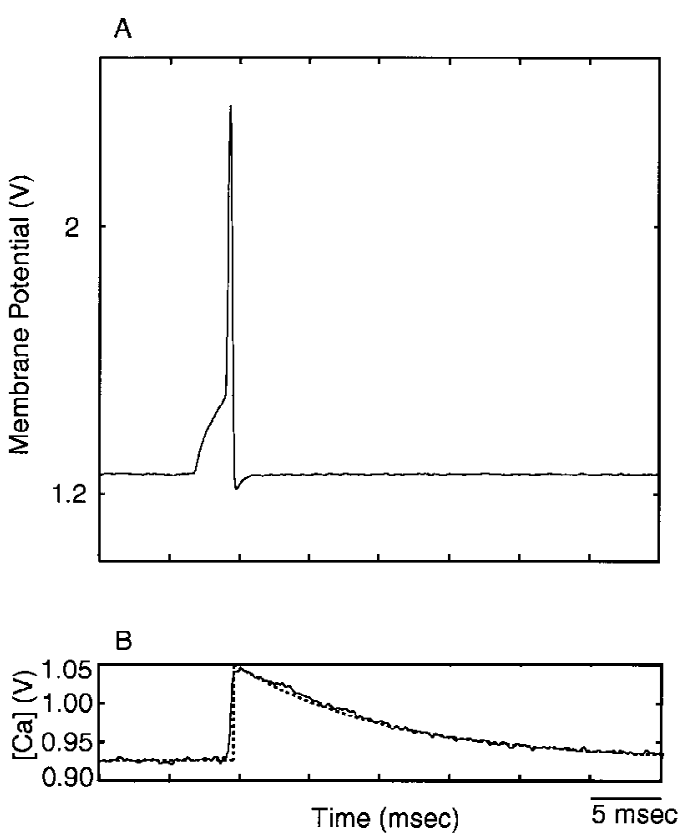

Figure 2: Impulse response of the calcium buffering and decay function. (A) Single action potential evoked by a brief current input. (B) The impulse response of the intracellular calcium concentration following this spike (solid line). $\mathrm{Ca}^{2+}$ ions are assume to enter via high-threshold, voltage-dependent calcium channels. The dashed line corresponds to the impulse response $h(t)=u(t) 0.12 e^{-t / \tau_{C a}}$ with a calcium decay time constant $\tau_{C a}=15 \mathrm{msec}$.

by a factor of $1 / \sqrt{2}$, is $f_{c}=1 /\left(2 \pi \tau_{C a}\right)$. We set $\tau_{C a}=15 \mathrm{msec}$ in the silicon neuron, making the cutoff frequency about $11 \mathrm{~Hz}$. Past this frequency, the filter drops off approximately as $1 / f$.

Let

$$
y(t)=\sum_{i} \delta\left(t-t_{i}\right)
$$

be the train of spikes where the $t_{i}$ 's denote the occurrence times of spikes in response to the stimulus, $s(t)$ (here the somatic current). Given a spike train, the original input signal can be reconstructed (or estimated) in a least-square sense using a reconstruction filter $K(t)$ that can be computed by well-known techniques (Rieke et al., 1996; Gabbiani and Koch, 1998),

$$
s_{r}(t)=\int K\left(t-t^{\prime}\right) y\left(t^{\prime}\right) d t^{\prime}
$$




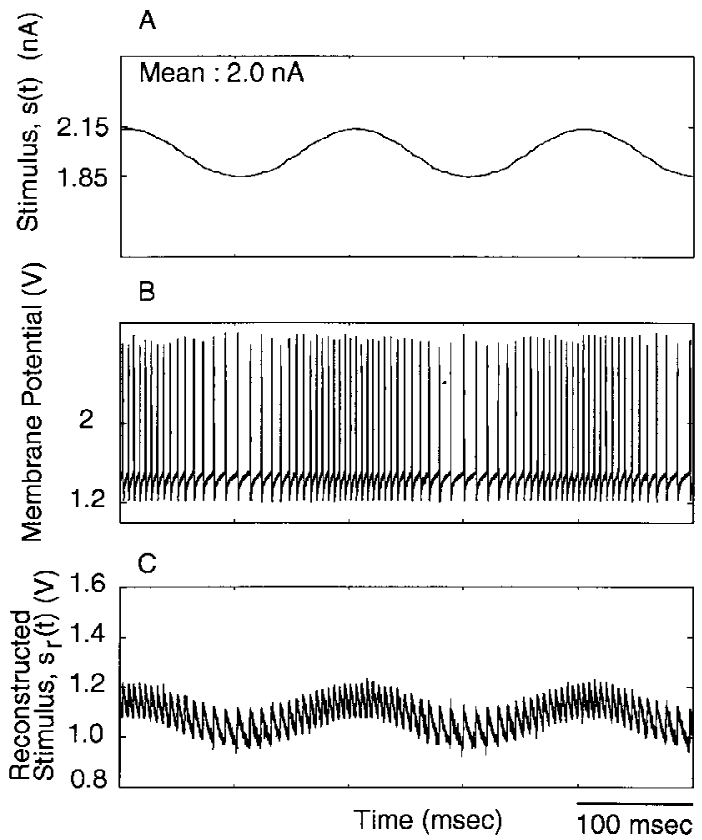

Figure 3: Basic behavior of the silicon neuron. (A) A sinusoidal $5 \mathrm{~Hz}$ current signal $s(t)$ is injected into the somatic compartment, with mean of $2.0 \mathrm{nA}$ and a peak-to-peak magnitude of $0.3 \mathrm{nA}$. (B) The cell generates spikes in response to this input. (C) The intracellular calcium concentration $\left[\mathrm{Ca}^{2+}\right]_{i}$ reflects the dynamics of the input current. Indeed, this biophysical variable can be thought of as an estimate $s_{r}(t)$ of the input if the input does not change too rapidly.

We here approximate this optimal reconstruction filter by the first-order low-pass filter implemented by $\left[\mathrm{Ca}^{2+}\right]_{i}$ and equate $K(t)$ with the impulse response $h(t)$ of equation 3.1 (see also Figure 2B).

Figure $3 \mathrm{C}$ shows how the low-pass filtering of spikes via intracellular calcium accumulation in our silicon neuron reconstructs a $5 \mathrm{~Hz}$ sinusoidal input signal. The spectrum of the associated calcium signal (see Figure 4A) illustrates that this input signal can easily be identified.

From frequency spectra such as these, we compute the signal-to-noise ratio (SNR) as the ratio of the magnitude of the signal peak, corresponding to the input modulation frequency, to the background noise level around this peak (Oppenheim \& Schafer, 1975; Irons, 1986). This number, expressed in decibels, is then

$$
\mathrm{SNR}=20 \log _{10} \text { magnitude ratio }=10 \log _{10} \text { power ratio. }
$$

In the case of Figure $4 \mathrm{~A}$, the SNR is $30 \mathrm{~dB}$. 

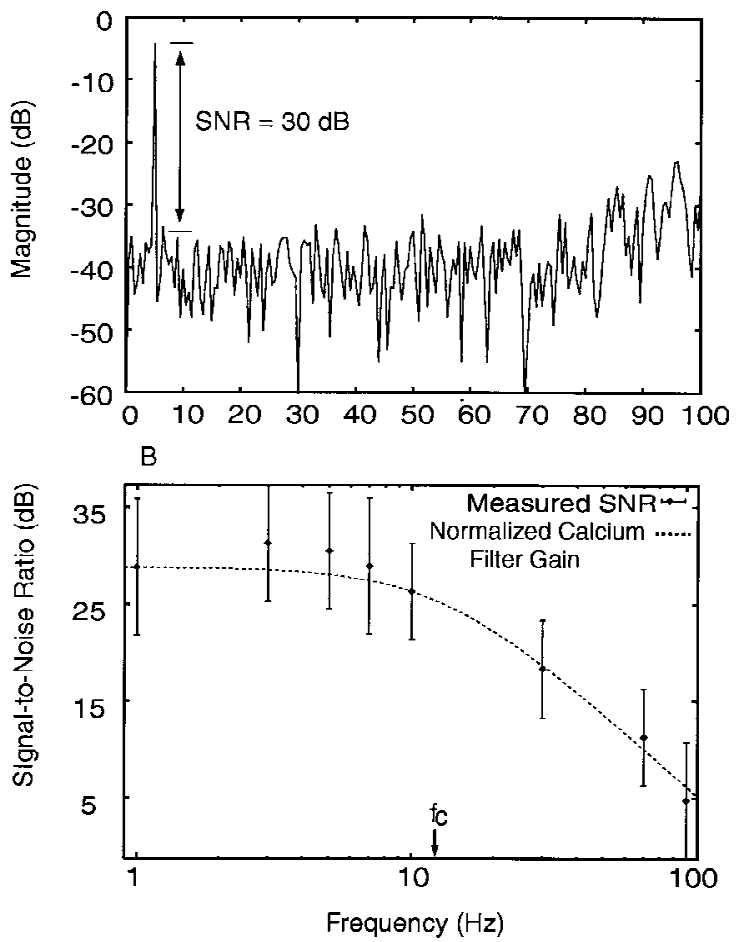

Figure 4: (A) The frequency spectrum representation of $\left[\mathrm{Ca}^{2+}\right]_{i}$ following injection of a sinusoidal $5 \mathrm{~Hz}$ signal (see Figure 3). The signal can clearly be identified. We can compute a signal-to-noise ratio (SNR) as the ratio of the peak response at the input frequency (signal) to the background noise level around this peak. In this case, SNR is about $30 \mathrm{~dB}$. (B) Measured SNR when the frequency of the input is swept between 1 and $100 \mathrm{~Hz}$, while the amplitude of the signal is maintained. The dashed line corresponds to the normalized gain characteristic associated with the low-pass filter of equation 3.1 with $\tau_{C a}=15 \mathrm{msec}$. The arrow points to the corner frequency $f_{c}$ where the gain is reduced by $1 / \sqrt{2}$.

Figure $4 \mathrm{~B}$ shows the dependence of the SNR on the frequency of the sinusoidal current injected into the soma for frequencies between 1 and $100 \mathrm{~Hz}$ while maintaining the same magnitude of the sinusoidal somatic current signal. For the region beyond the corner frequency of $11 \mathrm{~Hz}$, the SNR is inversely proportional to the frequency. Thus, the input signal can be relatively faithfully reconstructed (provided the input signal is above the current threshold necessary to evoke an action potential) for band-limited somatic current signals up to $11 \mathrm{~Hz}$. At higher frequencies, the reconstruction error-that is, the difference between the original signal $s(t)$ and its estimate $s_{r}(t)$-becomes larger. 
As a result, we can use the low-pass filtered response of the spike-evoked $\mathrm{Ca}^{2+}$ signal to monitor the time-varying somatic signal for our adaptation process described next.

\section{Adaptive Neural Coding}

A common way to represent the input-output characteristic of the neural spike encoding process is by its discharge, current frequency, or $\mathrm{f}-\mathrm{I}$ curve in response to somatic current steps. The dynamic range of the input can then be defined as the firing frequency range $\Delta f\left(f_{1} \leq f \leq f_{2}\right)$ over which a change in the input leads to a proportional change in the neuron's output frequency.

Given the maximum input activation of any particular neuron, it should possess a very large dynamic input range as well as high sensitivity to small differences in input signal. However, these are to some extent mutually exclusive goals. Maximizing sensitivity implies maximizing the slope of the $\mathrm{f}-\mathrm{I}$ curve, while maximizing the dynamic range implies minimizing the slope. Figure 5A shows an $\mathrm{f}-\mathrm{I}$ curve that defines a large input dynamic range. However, it has a low sensitivity to small-magnitude current signals.

One way in which the input dynamic range can be maximized without losing sensitivity is a steep $\mathrm{f}-\mathrm{I}$ curve with a fairly narrow input dynamic range whose operating point is shifted (see Figure 5B). This is accomplished by tracking the input over time and shifting the operating range of the $\mathrm{f}-\mathrm{I}$ curve to match the level of the mean or d.c. component of the signal. If the average input current is low, the neuron operates with f-I curve I (see Figure 5B). For increasing mean input levels, the f-I curve shifts to II and III. This scheme achieves a constant and high degree of sensitivity and a large operating range at a price: it takes time to adapt the $\mathrm{f}-\mathrm{I}$ curve to the mean stimulus current.

But why not also adapt the slope of the $\mathrm{f}-\mathrm{I}$ curve to the dynamic range of the input signal? If the dynamic range of the input signal in time is high, the slope should be shallow, maximizing the dynamic range of the f-I curve, while an input signal with a small, dynamic range optimizes the SNR of the output if the f-I curve becomes steeper (see Figure 5C). If both the mean and the dynamic range of input signals change simultaneously, the f-I curve needs to adapt its characteristic to match the change of the signal magnitude range (see Figure 5D).

We foundthat changing the leak conductance, $\bar{g}_{\text {leak }}$, shifts the f-I curve associated with our silicon neuron horizontally while maintaining its slope (increasing $\bar{g}_{\text {leak }}$ offsets the injected current, thereby increasing the current threshold needed to fire). Contrariwise, the amplitude of the peak calciumdependent potassium conductance $\left(\bar{g}_{A H P}\right)$ adjusts the slope of the f-I curve while maintaining the same current threshold. Increasing $\bar{g}_{A H P}$ increases the amount of afterhyperpolarization, causing multiple spikes to be spaced out further, but does not affect the initial threshold for spiking (Yamada, Koch, 

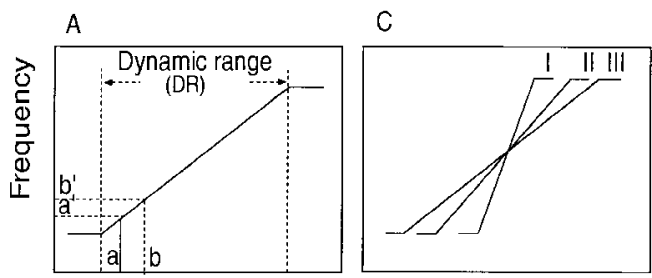

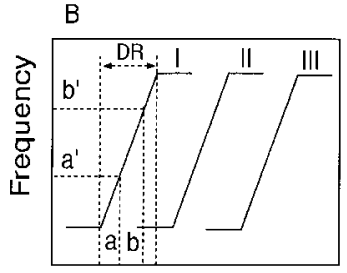

Current
D

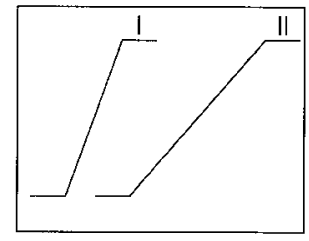

Current

Figure 5: Relationship between the current-frequency (f-I) curve of an idealized cell, its gain, and its dynamic input range. (A) An f-I curve that shows a largeinput dynamic range. This seems to be an ideal input dynamic range for the neuron because it is large enough to handle large-input current dynamic range. Unfortunately, to achieve this large dynamic range, sensitivity (or gain) must be sacrificed since maximal sensitivity implies an arbitrarily steep f-I curve. Since the total frequency range of any neuron is limited from below by zero and from above by saturation, this limits the dynamic range. The obvious solution to this dilemma can be solved by a steep f-I curve with a fairly narrow dynamic range whose operating point can be shifted (B). For increasing mean input levels, the f-I curve shifts to II and III. This scheme achieves high sensitivity and a large operating range, but at a price: it takes time to adapt the $\mathrm{f}-\mathrm{I}$ curve to the mean stimulus current (C). However, if the dynamic range of the input signal in time is high, the slope should be shallow, maximizing the dynamic range of the $\mathrm{f}-\mathrm{I}$ curve, while an input signal with a small dynamic range will optimize the SNR of the output if the $\mathrm{f}-\mathrm{I}$ curve becomes steeper (D). If both the mean and the dynamic range of input signals change simultaneously, the $\mathrm{f}-\mathrm{I}$ curve needs to adapt its characteristic to match the change of the signal magnitude range.

\& Adams, 1989). Changing both $\bar{g}_{\text {leak }}$ and $\bar{g}_{A H P}$ changes both the current threshold and the gain of the f-I curve.

Our adaptive neural coding procedure is described by

$$
\tau_{\text {adapt }} \frac{d \bar{g}_{\text {leak }}}{d t}=-\bar{g}_{\text {leak }}+K_{2} \theta\left(\min \left(s_{r}(t)\right)-a\right)+K_{1},
$$

and

$$
\tau_{\text {adapt }} \frac{d \bar{g}_{A H P}}{d t}=-\bar{g}_{A H P}+K_{2} \theta\left(\max \left(s_{r}(t)\right)-b\right)+K_{1} .
$$


Here $a>$ CAREF, $b>a, \theta(\cdot)$ is the Heaviside function, the min and max are evaluated within a suitable time window, $\tau_{\text {adapt }}$ is the time constant associated with adaptation, and $K_{1}$ and $K_{2}$ determine the lower (positive) and upper range of the two dynamic variables, $\bar{g}_{\text {leak }}$ and $\bar{g}_{A H P}$. In principle $\tau_{\text {adapt }}, K_{1}$, and $K_{2}$ can differ for the two conductances, yet we equate them here for convenience.

As long as the initial values of $\bar{g}_{\text {leak }}$ and $\bar{g}_{A H P}$ are chosen such that they satisfy $K_{1} \leq \bar{g}_{\text {leak }} \leq K_{1}+K_{2}$ and $K_{1} \leq \bar{g}_{A H P} \leq K_{1}+K_{2}$, these equations restrict $\bar{g}_{\text {leak }}$ and $\bar{g}_{A H P}$ to lie between $K_{1}$ and $K_{1}+K_{2}$.

We approximate the minimum of the reconstructed signal by computing the average calcium concentration $\mu$ minus its standard deviation $\sigma$, that is,

$$
\min \left(s_{r}(t)\right) \approx \mu-\sigma,
$$

and the maximum of the reconstructed signal by the mean plus one standard deviation,

$$
\max \left(s_{r}(t)\right) \approx \mu+\sigma .
$$

$\mu$ and $\sigma$ are running estimates of the mean and the standard deviation with

$$
\mu=\frac{1}{T} \int_{t-T}^{t}\left[C a^{2+}\right]_{i}(t) d t,
$$

and

$$
\sigma^{2}=\frac{1}{T} \int_{t-T}^{t}\left(\left[C a^{2+}\right]_{i}(t)-\mu\right)^{2} d t .
$$

Other ways of estimating the maximum and minimum of the estimated signal, such as the peak-to-peak magnitude, the envelope of the signal, and so on, are possible (see Liu, Golowasch, Marder, \& Abbott, 1998).

The adaptation algorithm works as follows. If $\mu+\sigma$ is above (resp. below) a high-calcium threshold, $b$, the total amount of calcium-dependent potassium conductance-that is, the density of the underlying channelsis increased (resp. decreased). Increasing this conductance affects the slope of the cell's discharge curve but not its intercept, thereby enlarging the input dynamic range of the $\mathrm{f}-\mathrm{I}$ curve. Conversely, if $\mu-\sigma$ is above (resp. below) a low calcium threshold, $a$, the total amount of leak conductance is increased (resp. decreased). Changing the leak conductance affects the current threshold for spiking but not the slope of the $\mathrm{f}-\mathrm{I}$ curve.

Varying both $\bar{g}_{\text {leak }}$ and $\bar{g}_{A H P}$ changes both current threshold and gain of the $\mathrm{f}-\mathrm{I}$ curve, resulting in adaptation to nonstationary arbitrary somatic current signals.

As long as the f-I curve increases monotonically and the leak and calciumdependent potassium conductances are adjusted independently of each other, this negative feedback, adaptive neural coding procedure always con- 
verges to an optimum. Within the range of $\bar{g}_{\text {leak }}$ and $\bar{g}_{A H P}$ values used, this optimum is a global one (since the relationship between $\bar{g}_{\text {leak }}$ and the intercept of the $\mathrm{f}-\mathrm{I}$ curve is a monotonic one, as is the relationship between $\bar{g}_{A H P}$ and the slope of the f-I curve).

Since we are primarily interested in adapting neurons to slow changes in input and in ambient operating conditions, our adaptive neural coding procedure can operate continuously while the neuron is transforming nonstationary somatic current signals into spike trains in its normal mode and does not require a separate, offline learning mode.

Note that our algorithm does not, in general, maximize the cell's SNR. Unless the metabolic cost of spiking is incorporated into such an optimization scheme, it could lead to very high firing rates. Rather, our algorithm enables the neuron's spiking characteristics to match optimally-over a long timescale-the mean and the variance of the input to the cell's firing characteristics.

\section{Results}

We applied this algorithm to our silicon neuron in its standard parameter settings (see Figure 1). For any given input injected into the somatic compartment, a digital computer senses $\left[\mathrm{Ca}^{2+}\right]_{i}$-the estimate of the reconstructed signal $s_{r}(t)$-from the silicon neuron and numerically computes $\mu$ and $\sigma$ over a 1-second-long time period. The low-threshold $a$ was set to $1.0 \mathrm{~V}$ and $1.3 \mathrm{~V}$ for the high-threshold $b$ (see Figure 1B). The bias voltages expressing $\bar{g}_{\text {leak }}$ and $\bar{g}_{A H P}$ were updated appropriately every 1 second by a minimal amount of $1.2 \mathrm{mV}$ ( $\bar{g}_{\text {leak }}$ and $\bar{g}_{A H P}$ were controlled by bias voltages ranging from 0 to $0.6 \mathrm{~V}$ with this resolution).

We used low-pass filtered random signals to test the adaptive neural coding principle. Figure 6A illustrates such a signal with a mean of $2 \mathrm{nA}$ and a standard deviation of $0.2 \mathrm{nA}$ (generated by filtering white noise using a second-order low-pass filter with a cutoff frequency of $8 \mathrm{~Hz}$ ), Figure $6 \mathrm{~B}$ the associated spike train, and Figure $6 \mathrm{C}$ the reconstructed signal from spikes. The mean of the reconstructed signal $s_{r}(t)$ was $1.15 \mathrm{~V}$, with a standard deviation of $0.15 \mathrm{~V}$.

For testing adaptation to the mean, we changed the mean current from $2 \mathrm{nA}$ to $2.5 \mathrm{nA}$ and $3.3 \mathrm{nA}$, maintaining the same standard deviation. The resultant $\mathrm{f}-\mathrm{I}$ curves after adaptation was complete are plotted in Figure 7A. For these curves, $\bar{g}_{\text {leak }}$ changed from a baseline value of $1.27 \mathrm{nS}$ to 2.03 and $2.77 \mathrm{nS}$, respectively.

Adaptation took 12 seconds to effect the shift from curve I to II and 23 seconds to shift from curve I to III. Provided that the associated time constants of adaptation are large enough to be able to sample a number of interspike intervals, they can be set to different values (we here use 1 second). For any fixed setting, adaptation takes longer if the shift in average input current is larger. 


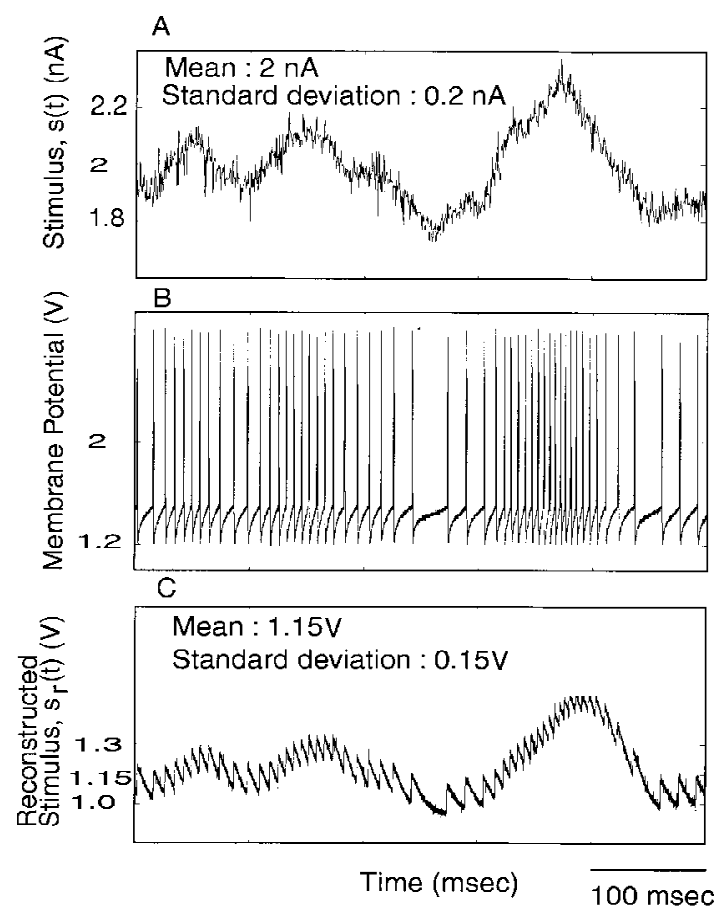

Figure 6: Response of the silicon neuron to a random input current-here a second-order low-pass (cutoff frequency of $8 \mathrm{~Hz}$ ) filtered random current signals with $2.0 \mathrm{nA}$ mean and $0.2 \mathrm{nA}$ standard deviation. These are the types of signals we used to evaluate our adaptive neural coding procedure. (B) The resultant spike train and $(\mathrm{C})$ somatic calcium concentration (with a mean of $1.15 \mathrm{~V}$ and a standard deviation of $0.15 \mathrm{~V})$.

In order to evaluate adaptation to the dynamic range of the input, we increased the standard deviation of the random signals (while maintaining the same mean current of $2 \mathrm{nA}$ ) from its base level of $0.2 \mathrm{nA}$ by adjusting both $\bar{g}_{\text {leak }}$ and $\bar{g}_{A H P}$. In its baseline state (curve I in Figure $\left.7 \mathrm{~B}\right), \bar{g}_{\text {leak }}=1.27 \mathrm{nS}$ and $\bar{g}_{A H P}=2.0 \mathrm{pS}$. Increasing the standard deviation of the random signal to $0.25 \mathrm{nA}$ (resp. $0.3 \mathrm{nA}$ ) causes a shift in $\bar{g}_{\text {leak }}$ to $1.13 \mathrm{nS}$ (resp. $0.97 \mathrm{nS}$ ) and an increase in $\bar{g}_{A H P}$ of $8.5 \mathrm{pS}$ (resp. $18 \mathrm{pS}$ ) (corresponding to curves II' and III' in Figure 7B, respectively).

What occurs when the somatic input signal is subthreshold, that is, too weak to evoke a spike? This is the scenario treated in Figure 8. The intracellular calcium concentration converges under this condition to the resting calcium concentration, CAREF (see Figure 8A). Since the low calcium threshold $a$ is set to a value larger than CAREF, the second term in equation 4.1 is 

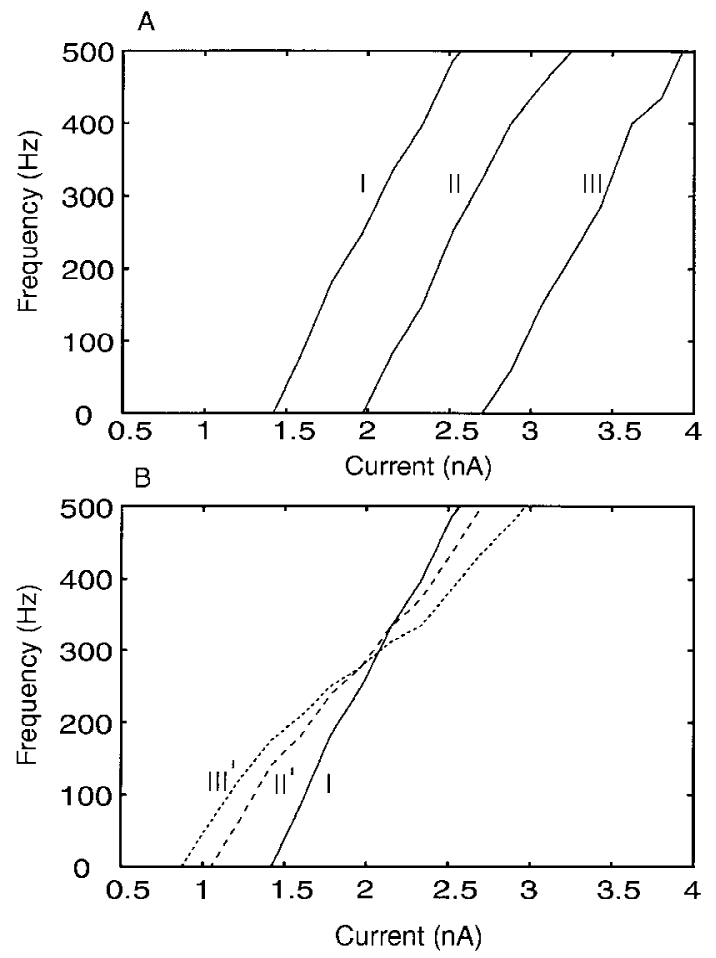

Figure 7: Adaptation of our silicon neuron in response to random signals of the type illustrated in Figure 6 whose mean (A) or standard deviation (B) was changed. The original $\mathrm{f}-\mathrm{I}$ curve of the neuron using its standard settings is labeled curve I in both panels. (A) The mean of the input (shown in Figure 6A) was increased from $2.0 \mathrm{nA}$ to 2.5 and $3.3 \mathrm{nA}$. Our algorithm adapts to these increased levels of input current by changing the maximum leak conductance, resulting in a shift in the steady-state discharge curve (from I to II and III in 12 respectively 23 seconds). This keeps the averaged firing rate constant. (B) In a second experiment, the standard deviation of the input was changed from $0.2 \mathrm{nA}$ to 0.25 and $0.3 \mathrm{nA}$, while its mean value was maintained at $2.0 \mathrm{nA}$. Our algorithm responded to this increase by decreasing the gain of the steady-state f-I curve (by increasing $\bar{g}_{A H P}$ ) while simultaneously shifting the intercept of the f-I curve to lower values (by decreasing $\bar{g}_{\text {leak }}$ ). The system reached its new equilibrium curve II' after 29 seconds and curve III' after 45 seconds.

zero and, since $K_{1}<\bar{g}_{\text {leak }}$, the right hand will be negative. In other words, the membrane leak conductance decreases until the neuron starts to fire (see Figures $8 \mathrm{~B}, 8 \mathrm{C}$, and $8 \mathrm{D}$ ) or until it reaches a minimum at $K_{1}$ (see Figure $8 \mathrm{E}$ ). The final firing rate following completion of adaptation (see Figure 8D) is 


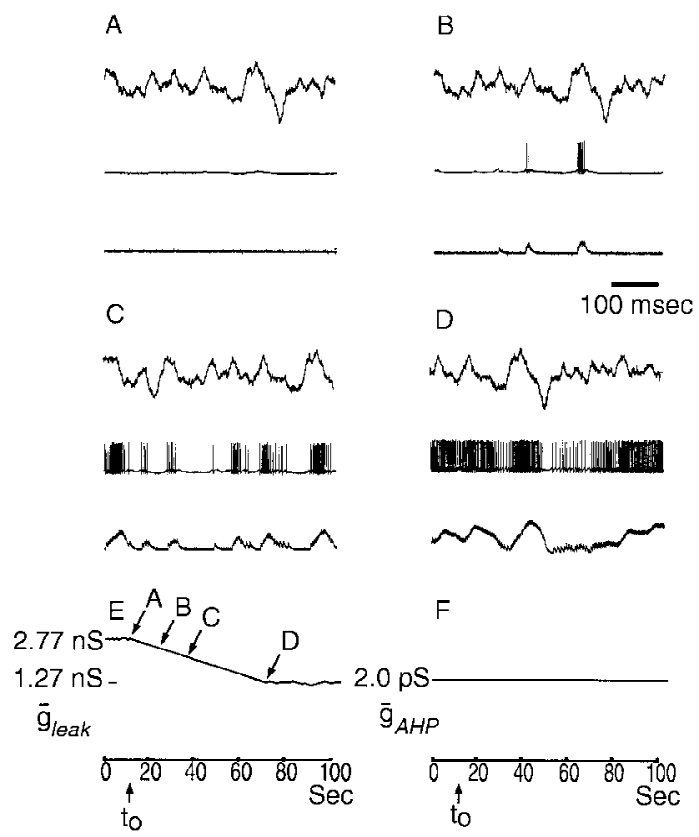

Figure 8: Adaptation to a subthreshold input. Initially, the f-I curve of the neuron was set to curve III in Figure $7 \mathrm{~A}\left(\bar{g}_{\text {leak }}=2.77 \mathrm{nS}\right.$ and $\left.\bar{g}_{A H P}=2.0 \mathrm{pS}\right)$, and a random signal with mean of $3.3 \mathrm{nA}$ and standard deviation of $0.2 \mathrm{nA}$ was injected. Each panel shows the random input signal (top), the membrance potential (middle), and $\left[\mathrm{Ca}^{2+}\right]_{i}(t)$ (top). (A) At $t_{o}$ the mean of the random signal was reduced to a subthreshold value of $2 \mathrm{nA}$, causing $\left[\mathrm{Ca}^{2+}\right]_{i}$ to drop to CAREF. Our adaptation algorithm leads to a slow reduction in $\bar{g}_{\text {leak }}$, following equation 4.1. After 11 seconds (B), the neurons start spiking, firing vigorously after $20 \mathrm{sec}-$ onds (C). The firing dynamics of the cell has converged after about 60 seconds to something close to curve I in Figure 7A (panel D). (E) The evolution of $\bar{g}_{\text {leak }}$ that causes the shift in the cell's f-I curve. (F) Since $K_{1}$ was set to the minimal value of $\bar{g}_{A H P}=2.0 \mathrm{pS}$, no change in this conductance occurs (see equation 4.2).

controlled by numerous parameters such as the two calcium thresholds, $a$ and $b$, and so on.

Because we wanted to demonstrate that subthreshold adaptation can be accomplished by shifting the $\mathrm{f}-\mathrm{I}$ curve rather than by adjusting the slope of the f-I curve, $K_{1}$ was set to the value of $\bar{g}_{A H P}$, and no change in this variable occurs (see Figure 8F).

In addition to the random current signal illustrated here, we also employed sinusoidal current signals with adjustable offsets to confirm that 
our algorithm adapts the firing behavior of our cell to match optimally the first and second moment of the input current (not shown).

\section{Discussion}

In this study we investigate an efficient adaptive algorithm that could plausibly be implemented at the single-cell level using the concentration of free intracellular calcium. Specifically it adapts the firing behavior of a spiking neuron to reflect optimally both the mean and the variance of the input signal—current delivered to the cell body.

The basic assumption underlying our algorithm is that one can estimate the input signal, the current $s(t)$ at the soma delivered by an intracellular electrode or by synaptic input from the dendrites, from the resultant spike train (provided that this current is above threshold). Signal estimation theory provides us with the optimal (in the least square sense) filter that allows us to reconstruct $s(t)$ from the spike train (Rieke et al., 1996; Gabbiani \& Koch, 1998). We argue that the intracellular concentration of free $\mathrm{Ca}^{2+}$ approximates such a low-pass reconstruction filter. Note that we are not arguing that the function of the cell is explicitly to reconstruct its time-varying somatic current signal but that $\left[\mathrm{Ca}^{2+}\right]_{i}$ at the cell bodyreflecting the time-varying somatic current signal—can be used to adapt the cell.

In the presence of a high-threshold, voltage-dependent calcium conductance at the soma, each action potential causes an inrush of $\mathrm{Ca}^{2+}$ ions that diffuse throughout the intracellular compartment, bind to various enzymes, buffers, or intracellular organelles, or are pumped out again. As witnessed by Figure $2 \mathrm{~B}$ this can be reasonably well approximated in our silicon neuron by an exponential decay process. As inspection of Figure 4B makes clear, $\left[\mathrm{Ca}^{2+}\right]_{i}$ can be thought of as the reconstructed signal $s_{r}(t)$, provided that the input $s(t)$ does not change too rapidly and as long as $s(t)$ is above the threshold for action potential generation. This timescale is ideal to compensate for relatively slow environmental changes, such as temperature, cell growth, channel turnover, and so on, that affect neuronal performance. As $s(t)$ begins to change more rapidly, that is, contains significant energy above the corner frequency of $11 \mathrm{~Hz}$, the squared difference between the signal and its reconstruction increases.

The algorithm uses the reconstructed signal in the guise of $\left[\mathrm{Ca}^{2+}\right]_{i}$ to change the intercept and the slope of the cell's discharge curve to provide an optimal match between the input range of the signal and the firing characteristic of the neuron. Specifically, we vary $\bar{g}_{\text {leak }}$ to compensate for changes in the mean input current and $\bar{g}_{A H P}$ to compensate for changes in the standard deviation (or the contrast) of $s(t)$. This method works very well for our silicon neuron implemented in CMOS VLSI circuit technology (e.g., see Figure 7). It even allows the cell to adapt to a subthreshold current input (see Figure 8). 
To what extent real neurons vary the shape and slope of their f-I curve in response to a change in their electrical makeup, stimulus ensemble, or environment is only now beginning to receive attention from experimentalists. Studies investigating contrast adaptation and long-term changes in response to a general increase or decrease in cortical excitability have tended to emphasize the contribution of (pre)synaptic mechanisms (Mclean \& Palmer, 1996; Carandini \& Ferster, 1997; Turrigiano et al., 1998). Although it is known that retinal neurons can adapt to a change in the variance of a visual signal (Smirnakis et al., 1997) the underlying cellular mechanism remains unknown.

Ongoing experiments directly relevant to our proposed algorithm come from the laboratory of Turrigiano (Desai, Nelson, \& Turrigiano, 1998; Desai, Rutherford, \& Turrigiano, 1999). Blocking all spiking activity for two days in cultured neocortical pyramidal cell leads to a reduction in the spiking threshold, as well as a highly significant increase in the slope of the cell's f-I curve. This was - at least partially - caused by an increase in the fast sodium current and a decrease in certain potassium currents. It would be exciting if it could be shown that these changes in ionic currents optimize the cell's gain and dynamic range.

Our proposed adaptation mechanism will be inactivated by either blocking the high-threshold, voltage-dependent calcium current or by preventing the cell from firing-for instance, by application of TTX.

Following the pioneering work of Abbott and LeMasson (1993) and LeMasson et al. (1993), we link changes in the intracellular calcium concentration to the densities with which various ionic channels are expressed across the somatic membrane. Such a pathway is likely to be exceedingly complex and will involve calcium-sensitive genes critical for slow neuronal adaptive responses (Koutalos \& Yau, 1996; Ginty, 1997). Our algorithm assumes that some combination of biophysical and biochemical mechanisms exists that effectively estimates the minimal and maximal levels of $\left[\mathrm{Ca}^{2+}\right]_{i}-$ or its mean and standard deviation in our approximation-within some time window. How this could be implemented at the biophysical level remains an open problem.

It is also unclear how the presence of a significant amount of low-threshold, voltage-dependent calcium conductance at the soma and spike initiation zone will affect the estimate of the input current. Such a conductance will tend to confound the link between spiking activity and intracellular calcium concentration since it can be active below threshold in the absence of spiking.

The work described here also has implications for the design and fabrication of networks of electronic silicon neurons. As with their biological counterparts, silicon neurons have a very large number of associated parameters that need to be set properly in order for the cell to function properly. Furthermore, the performance of such neurons needs to be stable in the face of fluctuations of bias voltages, operating temperature, transistor mismatch, 
and spatial parameter variations across the chip. Finally, the sensitivity of these neurons should also reflect changing input statistics. We here show how a continuously operating feedback circuit can keep the cell adjusted to make maximal use of its limited bandwidth and sensitivity.

In the version of the adaptation algorithm reported here, computing the mean and standard deviation of the calcium signal was carried out on an external computer. We are currently designing a single chip that would contain the silicon neuron in addition to all circuitry necessary to perform the adaptation in situ on the basis of two different and complementary approaches. One analog circuit uses switched-capacitor memory (Elias, Northmore, \& Westerman, 1997) and the other floating-gates learning synapses (Diorio, Hasler, Minch, \& Mead, 1996) as the critical components to control the time constants of the adaptation process. This latter technology should enable us to design high-density, robust, and adaptive electronic neurons.

\section{Appendix}

The model we emulated using the silicon neuron has five active ion currents as well as a leak current that are engaged in the neural spike encoding process at soma (Douglas \& Mahowald, 1998). The membrane voltage $V$ at the soma is described by

$$
C_{m} \frac{d V}{d t}+I_{N a}+I_{K D}+I_{\text {leak }}+I_{A}+I_{C a}+I_{A H P}+I_{\text {Inject }}=0,
$$

where $I_{N a}$ represent the fast sodium current, $I_{K D}$ the delayed rectifier potassium current, $I_{A}$ a transient, inactivating potassium current, $I_{A H P}$ a calciumdependent potassium current, $I_{C a}$ a high-threshold calcium current, $I_{\text {leak }}$ the leakage current, and $I_{\text {Inject }}$ the current delivered to the cell body.

The leak current is given by $I_{\text {leak }}=g_{\text {leak }}\left(V-E_{l e a k}\right)$, where $E_{l e a k}$ is the resting potential.

The four voltage-dependent currents, $I_{N a}, I_{K D}, I_{A}$, and $I_{C a}$, can be approximated by a Hodgkin-Huxley-like formalism,

$$
I(t)=\bar{g} \cdot m(t, V)^{i} \cdot h(t, V)^{j} \cdot(V-E),
$$

where $\bar{g}$ is the maximum conductance; $m$, the activation variable taken to the $i$ th power; $h$, the inactivation variable taken to the $j$ th power; and $E$, the associated reversal potential of the current. The dynamics of each activation and inactivation particle is governed by a first-order differential equation,

$$
\frac{d m}{d t}=\frac{m_{\infty}(V)-m}{\tau_{m}(V)}
$$

and

$$
\frac{d h}{d t}=\frac{h_{\infty}(V)-h}{\tau_{h}(V)},
$$


where $m_{\infty}(V)$ and $h_{\infty}(V)$ are the voltage-dependent activation curve and inactivation curve, and $\tau_{m}$ and $\tau_{h}$ are the time constants of the activation and the inactivation.

The afterhyperpolarization current, $I_{A H P}$, is a potassium current that depends on the intracellular calcium concentration $\left[\mathrm{Ca}^{2+}\right]_{i}$,

$$
I_{A H P}(t)=m_{A H P}\left(\left[C a^{2+}\right]_{i}\right) \cdot \bar{g}_{A H P} \cdot\left(V-E_{K}\right),
$$

where $E_{K}$ is the potassium reversal potential and $m_{A H P}$ the voltage-independent activation given by a sigmoidal function of the intracellular calcium concentration $\left[\mathrm{Ca}^{2+}\right]_{i}$,

$$
m_{A H P}=\frac{1}{1+\exp -\left(\left[\mathrm{Ca}^{2+}\right]_{i}-C A M T\right) / T} .
$$

Here CAMT is a constant determined by $(a+b) / 2(a$ is a low calcium threshold and $b$ a high calcium threshold) and $T$ is a parameter that determines the slope of the sigmoid.

Finally, the dynamics of free, intracellular calcium is governed by a single linear update expression,

$$
\tau_{C a} \frac{d\left[C a^{2+}\right]_{i}}{d t}=-\left[C a^{2+}\right]_{i}+\kappa I_{C a}+C A R E F,
$$

where $\tau_{C a}$ is a time constant of decay, $I_{C a}$ is the high-threshold calcium current, $\kappa$ is a constant that converts the incoming calcium current into a concentration change, and CAREF is the resting calcium concentration.

All parameters of this hardware model are under electronic control.

\section{Acknowledgments}

We thank Christoph Rasche, Dave Lawrence, and Brian Baker for their assistance. This work was supported by the Office of Naval Research, the Center for Neuromorphic Systems Engineering as a part of the National Science Foundation Engineering Research Center Program, the Swiss National Fund SPP programme, and a Colvin fellowship of the Division of Biology at Cal Tech to J. S.

\section{References}

Abbott, L. F., \& LeMasson, G. (1993). Analysis of neuron models with dynamically regulated conductances. Neural Computation, 5, 823-842.

Baddeley, R., Abbott, L. F., Booth, M. C. A., Sengpiel, F., Freeman, T., Wakeman, E. A., \& Rolls, E. T. (1997). Responses of neurons in primary and inferior temporal visual cortices to natural scenes. Proc. Roy. Soc. Lond. B, 264, 17751783 . 
Bayly, E. J. (1968). Spectral analysis of pulse frequency modulation in the nervous systems. IEEE Trans. Bio-Medical Engineering, BME 15, 257-265.

Bower, J. M., \& Beeman, D. (1995). The book of Genesis. New York: Springer-Verlag.

Calvin, W. H. (1978). Setting the pace and pattern of discharge: Do CNS neurons vary their sensitivity to external inputs via their repetitive firing processes? Federation Proc., 37, 2165-2170.

Carandini, M., \& Ferster, D. (1997). A tonic hyperpolarization underlying contrast adaptation in cat visual-cortex. Science, 276, 949-952.

Desai, N. S., Nelson, S. B., \& Turrigiano, G. G. (1998). Activity regulates intrinsic conductances in visual cortical neurons. Soc. Neurosci. Abstr.,

Desai, N.S., Rutherford, L. C., \& Turrigiano, G. G. (1999). Plasticity in the intrinsic electrical properties of cortical pyramidal neurons. Nature Neurosci., 2, 515520.

Diorio, C., Hasler, P., Minch, B. A., \& Mead, C. (1996). A single-transistor silicon synapse. IEEE Trans. Electron Devices, 43, 1972-1980.

Douglas, R. J., Koch, C., Mahowald, M., \& Martin, K. A. C. (1999). The role of recurrent excitation in neocortical circuits. In P. S. Ulinski, E. G. Jones \& A. Peters (Eds.), pp. 251-281. Cerebral cortex (Vol. 13). New York: Plenum.

Douglas, R., \& Mahowald, M. (1995). A construction set for silicon neurons. In S. Zornetzer et al. (Eds.), An introduction to neural and electronic networks (2nd ed.). New York: Academic Press.

Douglas, R., \& Mahowald, M. (1998). Design and fabrication of analog VLSI neurons. In C. Koch \& I. Segev (Eds.), Methods in neuronal modeling: From synapses to networks (2nd ed.) pp. 316-360. Cambridge, MA: MIT Press.

Elias, J. G., Northmore, D. P. M., \& Westerman, W. (1997). An analog memory circuit for spiking silicon neurons. Neural Computation, 9, 419440.

Fatt, P., \& Katz, B. (1951). An analysis of the end-plate potential recorded with an intracellular electrode. J. Physiol. (London), 115, 320-370.

Gabbiani, F., \& Koch, C. (1998). Principles of spike train analysis. In C. Koch \& I. Segev (Eds.), Methods in neuronal modeling: From synapses to networks (2nd ed.) pp. 316-360. Cambridge, MA: MIT Press.

Ginty, D. D. (1997). Calcium regulation of gene expression: Isn't that spatial? Neuron, 18, 183-186.

Hodgkin, A., \& Huxley, A. F. (1952). A quantitative description of membrane current and its application to conduction and excitation in nerve. J. Physiol. (London), 117, 500-544.

Irons, F. (1986). Dynamic characterization and compensation of analog to digital converters. IEEE Int. Symp. Circuits and Systems, 2, 1273-1277.

Jayant, N. S., \& Noll, P. (1984). Digital coding of waveforms. Englewood Cliffs, NJ: Prentice Hall.

Johnston, D., \& Wu, S. M. (1995). Foundations of cellular neurophysiology. Cambridge, MA: MIT Press.

Koch, C. (1998). Biophysics of computation: Information processing in single neurons. New York: Oxford University Press.

Koutalos, Y., \& Yau, K. (1996). Regulation of sensitivity in vertebrate rod photoreceptors by calcium. TINS, 19, 73-81. 
Laughlin, S. (1981). A simple coding procedure enhances a neuron's information capacity. Z. Naturforsch, 36c, 910-912.

LeMasson, G., Marder, E., \& Abbott, L. F. (1993). Activity dependent regulation of conductances in model neurons. Science, 259, 1915-1917.

Liu, Z., Golowasch, J., Marder, E., \& Abbott, L. F. (1998). A model neuron with activity-dependent conductances regulated by multiple calcium sensors. $J$. Neuroscience, 18, 2309-2320.

Mahowald, M., \& Douglas, R. (1991). The silicon neuron: A compact electronic analog that emulates the electrophysiological characteristics of real neurons. Nature, 354, 515-518.

Mclean, J., \& Palmer, L. A. (1996). Contrast adaptation and excitatory amino acid receptors in cat striate cortex. Visual Neuroscience, 13, 1069-1087.

Oppenheim, A., \& Schafer, R. (1975). Digital signal processing. Englewood Cliffs, NJ: Prentice Hall.

Partridge, L. D. (1965). Modifications of neural output signals by muscles: A frequency response study. J. Appl. Physiol., 20, 150-156.

Rieke, F., Warland, D., van Steveninck, R. R. D., \& Bialek, W. (1996). Spikes: Exploring the neural code. Cambridge, MA: MIT Press.

Shin, J. (1994). Generation mechanism of integrative potential in axon hillock of a single neuron and noise feedback pulse coding. World Congress on Neural Networks, IV, 391-396.

Shin, J. (1996). Roles of negative feedback potassium currents and recurrent inhibition. Soc. Neurosci. Abstr., 22, 793.

Shin, J., Lee, K., \& Park, S. (1993). Novel neural circuits based on stochastic pulse coding and noise feedback pulse coding. Int. J. Electronics, 74, 359-368.

Smirnakis, S. M., Berry, M. J., Warland, D. K., Bialek, W., \& Meister, M. (1997). Adaptation of retinal processing to image-contrast and spatial scale. Nature, $386,69-73$.

Turrigiano, G. G., Leslie, K. R., Desai, N. S., Rutherford, L. C., \& Nelson, S. B. (1998). Activity-dependent scaling of quantal amplitude in neocortical neurons. Nature, 391, 892-896.

van Steveninck, R. R. de R., Bialek, W., Potters, M., \& Calson, R. H. (1994). Statistical adaptation and optimal estimation in movement computation by the blowfly visual system. Proc. IEEE Int. Conf. on Systems, Man, and Cybernetics, 1, 302-307.

Widrow, B., \& Stearns, S. D. (1985). Adaptive signal processing. Englewood Cliffs, NJ: Prentice Hall.

Wong, P. W., \& Gray, R. M. (1990). Sigma-Delta modulation with i.i.d. gaussian inputs. IEEE Trans. Inf. Theory, 36, 784-798.

Yamada, W., Koch, C., \& Adams, P. (1989). Multiple channels and calcium dynamics. In C. Koch \& I. Segev (Eds.), Methods in neuronal modeling: From synapses to networks (pp. 97-133). Cambridge, MA: MIT Press.

Received December 1, 1997; accepted August 8, 1998. 